\title{
Domain-Domain Interaction Identification with a Feature Selection Approach
}

\author{
Xing-Ming Zhao and Luonan Chen \\ Institute of Systems Biology, \\ Shanghai University, 200444 Shanghai, China \\ xm_zhao@shu.eud.cn, chen@eic.osaka-sandai.ac.jp
}

\begin{abstract}
The protein-protein interactions (PPIs) are generally assumed to be mediated by domain-domain interactions (DDIs). Many computational methods have been proposed based on this assumption to predict DDIs from available data of PPIs. However, most of the existing methods are generative methods that consider only PPI data without taking into account non-PPIs. In this paper, we propose a novel discriminative method for predicting DDIs from both PPIs and non-PPIs, which improves the prediction reliability. In particular, the DDI identification is formalized as a feature selection problem, which is equivalent to the parsimonious principle and is able to predict both DDIs and PPIs in a systematic and accurate manner. The numerical results on benchmark dataset demonstrate that formulating DDI prediction as a feature selection problem can predict DDIs from PPIs in a reliable way, which in turn is able to verify and further predict PPIs based on inferred DDIs.
\end{abstract}

Keywords: Discriminative approach, domain-domain interaction, feature selection, protein-protein interaction.

\section{Introduction}

Proteins exert their functions by interacting with each other [1]. Generally, one protein interacts with its partner by binding one of its domains to the domain(s) in its target protein. In other words, proteins interact with each other through domaindomain interactions (DDIs) 2] 3]. Recently, many computational methods have been proposed to identify domain interactions from protein interactions. For example, Sprinzak and Margalit [4] proposed the Association method for predicting domain interactions based on the frequency of observed protein interactions that contain the pair of domains. Deng et al. 5] presented a maximum likelihood estimation (MLE) method as well as an Expectation-Maximization (EM) algorithm to infer underlying domain interactions from protein interactions. Liu et al. 6] combined protein interactions from multiple species to identify interacting domain pairs. Riley et al. [7] developed a new method, namely Domain Pair Exclusion Analysis (DPEA), to predict domain interactions based on all of the protein interactions from Database of Interacting Proteins (DIP) [8, where a new score, i.e. E-value, is developed to assess the contribution of each possible domain pair

M. Chetty, A. Ngom, and S. Ahmad (Eds.): PRIB 2008, LNBI 5265, pp. 178 186, 2008.

(C) Springer-Verlag Berlin Heidelberg 2008 
to the likelihood of a set of observed protein interactions. It is shown that the DPEA method outperforms both MLE [5] and Association method [4]. Recently, Guimaräes et al. 9] developed a linear programming model, namely Parsimonious Explanation (PE), to predict domain interactions based on the parsimonious principle with the assumption that a given set of protein-protein interactions are accomplished through the minimal set of domain interactions. The PE method is shown to outperform DPEA [7, Association [4] and MLE [5] based on numerical simulations. Moreover, Lee et al. [10] developed a Bayesian approach to predict high confidence domain interactions based on the integration of multiple data sources from multiple species, where the integration of multiple data sources significantly improves the prediction accuracy compared with single data source analysis.

The methods described above assume that protein interactions are mediated by domain-domain interactions, and they try to identify the DDIs underlying the PPIs. Despite the success on specific datasets, most of the existing computational methods are generative methods, where a model is constructed based on the protein-protein interaction data with the assumption that proteins interact with each other through domain-domain interactions. However, the existing methods use only available data of PPIs without the consideration of the non-PPIs, which may result in imbalance problem of information [1] [12. Since the proteins are assumed to interact through domain interactions, domain pairs occurring in the non-PPIs are more likely false DDIs [7. Therefore, the non-PPI data can provide insight into the domain interaction.

In this paper, we proposed a novel discriminative approach, namely domain interaction prediction in a discriminative way (DIDD), to predict domain interactions based on protein interactions. Different from the existing methods, both PPIs and non-PPIs are considered in DIDD, thereby not only alleviating the imbalance problem of information but also improving prediction accuracy. In particular, DDI prediction is formulated as a feature selection problem in machine learning, which is in consistent with parsimonious principle that protein interactions are accomplished through the minimum set of domain interactions. In feature selection, the possible domain pairs are assessed according to their contributions to the discrimination between PPIs and non-PPIs. The proposed method is able to predict DDIs based on PPIs, which in turn can predict and verify PPIs based on the inferred DDIs, i.e. selected features in this case. The numerical results on benchmark datasets demonstrate the effectiveness and efficiency of the proposed method.

The rest of the paper is organized as follows: Section 2 describes the methods that are proposed for identifying DDIs from PPIs; Section 3 presents the numerical results on benchmark dataset; The conclusions are drawn finally.

\section{Methods}

The idea behind DDI identification in PPIs is that PPIs are mediated by DDIs. Therefore, the domain pairs that best discriminate PPIs and non-PPIs are more 
likely the true DDIs. The PE 9 method assumes that the given protein interactions can be approximately accomplished by the minimum set of domain-domain interactions, which is in consistent with the idea behind feature selection that tries to find out as few informative features (e.g. DDIs) as possible for classification (e.g. discrimination between PPIs and non-PPIs). However, feature selection works in a discriminative way that take into account non-PPIs. Therefore, higher prediction accuracy is expected.

\subsection{Feature Vector Construction}

The discrimination between PPIs and non-PPIs is actually a binary classification problem. To utilize the discriminative methods, the positive samples (i.e. PPIs) and negative samples (i.e. non-PPIs) should be represented as feature vectors. In this work, each sample is a protein pair (either interacting pair or non-interacting pair) and is represented as a vector. The positive samples are PPIs from protein interaction database, whereas the negative samples are protein pairs that are randomly generated except the known PPIs. The rationality behind the method generating negative samples is that only one out of six hundred randomly generated protein pairs is possibly the true PPI, and this method has also been employed widely in the literature [13] 14. For the PPIs, all the possible combinations of two domains are found and kept in order, where each domain pair exists in at least one interacting protein pair. After getting all the domain pairs, each sample is represented as a feature vector, where the feature value is 1 if the corresponding domain pair occurs in the sample and otherwise 0 .

\subsection{Classifier}

After constructing the feature vectors, we need to design classifier to discriminate the PPIs from non-PPIs. It can be seen that the vectors generated above have following properties: 1) sparse content, i.e. most of the feature values are $0 ; 2$ ) high dimension due to the large number of possible combinations among domains; 3) few positive samples but large number of negative smaples. Therefore, the conventional classifiers such as Support Vector Machines and Nearest neighbor classifier cannot be used here.

In this paper, we designed a simple classifier to discriminate PPIs from nonPPIs based on the specific data structure and the assumption that DDIs mediate PPIs. For a given protein pair vector $\mathbf{x}_{\mathbf{i}}$, the class label $y_{i}$ corresponding to it can be defined as:

$$
y_{i}= \begin{cases}1, & \text { if } \sum_{j} x_{i j} \geq 1, \\ -1, & \text { otherwise, }\end{cases}
$$

where $x_{i j}$ is the value of the $j$ th feature in the $i$ th sample. The idea behind the classifier is that if the domain combination corresponding to $x_{i j}$ is the true DDI and the protein pair $\mathbf{x}_{\mathbf{i}}$ contains the domain combination (i.e. $x_{i j}=1$ in this case), then the protein pair $\mathbf{x}_{\mathbf{i}}$ is an interacting pair and $y_{i}=1$ accordingly. Since the classifier does not involve any model training procedure, there is not any problem of overfitting. 


\subsection{Feature Selection}

Given a set of PPIs and non-PPIs, we want to find out which domain combinations mediate the PPIs, i.e. the putative DDIs. With the constructed vectors and the assumption that PPIs are mediated by DDIs, we can formulate DDI prediction as a feature selection problem. In feature selection, the purpose is to find out as few informative features as possible to build a reliable and accurate learning model. In this case, feature selection aims to find out the domain pairs that discriminate PPIs from non-PPIs. It can be seen that the idea behind feature selection is equivelant to the parsimonious principle that the domain-domain interactions are well approximated by the minimum set of DDIs mediating the given set of PPIs [9. However, non-PPIs are also taken into account in this work. The domain pairs kept in feature selection are assumed to be the putative DDIs.

Considering the specific data structure and imbalance between positive and negative samples, the unbalanced correlation score proposed in [15] is utilized to rank the features, which is defined as:

$$
s_{j}=\sum_{y_{i}=1} x_{i j}-\lambda \sum_{y_{i}=-1} x_{i j}
$$

where $s_{j}$ is the score for the $j$ th feature, $y_{i}$ is the label for sample $\mathbf{x}_{i}, x_{i j}$ is the value for the $j$ th feature in vector $x_{i}$, and $\lambda$ is a penalty parameter to punish the occurrance of the feature in negative samples. Generally, a large value is adopted for $\lambda$, e.g. $\lambda=5$ in this work. The higher the score $s_{j}$ is, the higher feature $j$ is ranked. The idea behind the method is that the more frequently the feature occurs in positive samples and less in negative samples, the more informative it is, thereby more likely true DDIs.

\subsection{Performance Evaluation}

To see the performance of the classifier, the following measures are adopted in this work, including precision, recall, F1-measure:

$$
\begin{gathered}
\text { precision }=\frac{T P}{T P+F P} \\
\text { recall }=\frac{T P}{T P+F N} \\
F 1=\frac{2 * \text { precision } * \text { recall }}{\text { precision }+ \text { recall }}
\end{gathered}
$$

where $T P$ means the number of positive samples that are predicted correctly, $F N$ means the number of positive samples that are predicted as negative samples, $F P$ means the number of negative samples that are predicted as positive samples, and FP means the number of negative samples that are predicted correctly. 


\section{Results and Discussion}

To test the performance of the proposed method, the DIDD method was applied to predict DDIs based on a set of protein-protein interactions from 69 organisms, which was constructed by Riley and colleagues [7. This dataset is denoted as "Riley test set" here, and was also used by the PE method [9]. The Riley test set contains all the protein interactions from DIP database [8], and domains were assigned to proteins by employing the Pfam hidden markov model profiles [16]. Note that only Pfam-A domains were assigned to the proteins here. Table 1 lists the datasets used in this work, where PPI denotes protein-protein interactions, DDI denotes domain-domain interactions, proteins are proteins involved in PPIs, and number represents the number of PPIs, non-PPIs, proteins and potential DDIs used in this work.

Table 1. The datasets used in this work

\begin{tabular}{c|c|c|c|c}
\hline & PPIs & non-PPIs & Proteins & Potential DDIs \\
\hline Number & 26,032 & $11,651,400$ & 11,403 & 27,617 \\
\hline
\end{tabular}

The DIDD method was first applied to predict the DIP protein interactions by selecting informative features (i.e. domain pairs), where the unbalanced correlation score [15] was employed in feature selection. This procedure continues until the prediction accuracy does not improve any more. Consequently, the domain pairs corresponding to the selected features were seen as putative DDIs. With the iPfam dataset as the gold standard, we compared DIDD with previous methods, i.e. DPEA and PE methods, with respect to precision and recall, where the results by PE are those predicted with PPI reliability of $50 \%$ and pw-score $\leq 0.01$, and the top 3005 predictions by DPEA are seen as its predictions. For fair comparison, only the predictions by PE and DPEA that involve Pfam-A domains are considered. In particular, we investigated the number of difficult predictions by different methods as described in [9] and [7, where the necessity of assessing predictions with respect to the difficulty has been justified in [7. In this work, the definition of difficult prediction described in [9] was adopted to validate the proposed method, and a DDI is assumed to be difficult to predict if the domain pair is not contained in any single-domain interacting protein pair. Table 2 summarizes the prediction results by different methods on this dataset. From the results, we can see that the DIDD method outperforms the other two methods. Especially, DIDD can predict more difficult tasks compared with other two methods. The results clearly demonstrate the prediction power of the proposed method especially on difficult gold standard pairs, and thereby is a good complement to existing methods. Furthermore, the results also demonstrate that the non-PPIs can really help to improve the prediction accuracy.

In addition, to test the performance of DIDD, the datasets from DOMINE 17 database were employed to test how much of our predictions can be validated by at least one other existing computational method. DOMINE is a database that contains known and predicted domain interactions, including DDIs from 3DID 
Table 2. Comparison of different methods on DDI prediction based on Riley test set

\begin{tabular}{l|c|c|c}
\hline method & precision & recall & difficult predictions \\
\hline DPEA & $10.21 \%$ & $23.63 \%$ & 5 \\
PE & $12.21 \%$ & $29.63 \%$ & 75 \\
DIDD & $20.80 \%$ & $29.76 \%$ & 157 \\
\hline
\end{tabular}

Table 3. Comparison of various methods on Riley test set, where percentage means the percentage of predictions that can be validated by at least one other existing method.

\begin{tabular}{l|c|c|c|c}
\hline Methods & DIDD & DPEA+PE & RCDP & Bayesian \\
\hline Percentage & $50.71 \%$ & $23.80 \%$ & $39.60 \%$ & $41.50 \%$ \\
\hline
\end{tabular}

Table 4. Performance of various methods in predicting PPIs based on inferred DDIs for the Riley test set

\begin{tabular}{l|c|c|c}
\hline Methods & precision & recall & F1 \\
\hline DIDD & $100.00 \%$ & $6.07 \%$ & $11.44 \%$ \\
DPEA & $3.30 \%$ & $16.24 \%$ & $5.48 \%$ \\
PE & $3.68 \%$ & $19.19 \%$ & $6.17 \%$ \\
\hline
\end{tabular}

[18, iPfam [19] and those predicted by different computational approaches. In this example, the DIDD method was compared against DPEA [7, PE 9], RCDP [20] and Bayesian approach [10. Table 3] shows the comparison of various methods on how much of their prediction can be validated by at least one other existing computational method, where the statistics of the other methods are from the DOMINE database [17. From the results, we can see that most of our predictions have been validated by at least one other computational method and has higher prediction accuracy compared with other methods, which confirms the efficiency and effectiveness of DIDD. In the DOMINE [17] database, each predicted or known DDI is associated with a confidence score. To see the performance of DIDD, we further investigated how much of the our predictions have high confidence scores. Figure 1 shows the distribution of confidence scores for the predictions by DIDD, where we can see that a relatively small number of our predictions have low confidence scores, which clearly demonstrate the prediction power of DIDD.

In addition, to validate the predicted DDIs by the proposed method, we predicted PPIs based on the inferred DDIs. In addition, DIDD was compared against PE and DPEA in predicting PPIs based on the inferred DDIs to test the predicted DDIs. In this work, the classifier and samples (i.e PPIs and non-PPIs) used by DIDD were employed to test the predicted DDIs by PE [9] and DPEA [7, where the inferred DDIs were treated as the selected features as described in Methods. Table 4 shows the comparison of performance of various methods in predicting PPIs based on inferred DDIs. From Table 4 , it can be seen that the proposed DIDD method outperforms other existing methods in discriminating the PPIs and nonPPIs based on inferred DDIs. The DIDD method got high precision means that 


\section{Prediction results validated in DOMINE}

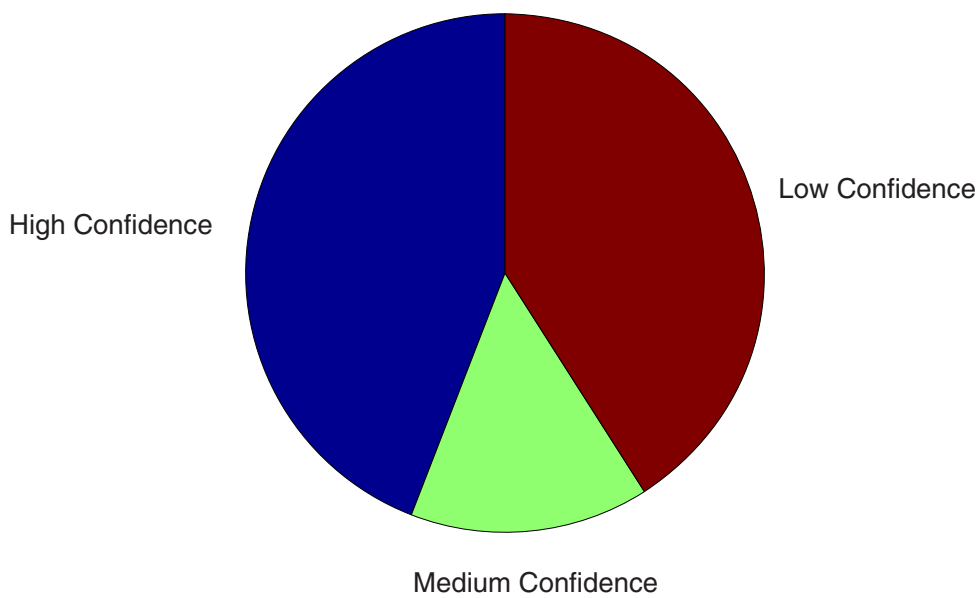

Fig. 1. Distribution of confidence scores for the predictions by DIDD on Riley test set

most of its predictions are true DDIs, while a low recall means that DIDD only predict a small number of domain pairs as domain interactions. The results demonstrate that the DDIs predicted by DIDD are more possibly the true DDIs that mediate PPIs compared against other existing methods, which confirms the effectiveness and efficiency of DIDD in predicting DDIs from PPIs. Furthermore, the DIDD can also validate and predict PPIs based on the inferred DDIs.

\section{Conclusions}

Understanding PPIs at domain level can provide insight into protein function and evolutionary history of PPIs. In this paper, a novel method, namely domain interaction prediction in a discriminative way (DIDD), is presented for predicting DDIs from data of available PPIs. Unlike existing methods, DIDD considers both PPIs and non-PPIs. Since PPIs are typically assumed to be mediated by DDIs, the domain combinations occurring in the non-PPIs are more possibly false DDIs. Therefore, higher prediction accuracy is expected for DIDD by taking non-PPIs into account. In particular, in this work, DDI prediction is formalized as feature selection, which is in consistent with parsimonious principle that DDIs can be approximated by the minimum set of DDIs that mediate the given PPIs [9]. By selecting the informative features, DIDD can predict those DDIs that really mediate the given PPIs, and in turn help to verify and predict PPIs based on the inferred DDIs. The results on benchmark data prove the predictive power of our method. In addition, the overlap between the predictions by DIDD and published results demonstrate the effectiveness and efficiency of the proposed method. 


\section{Acknowledgements}

This work was partly supported by the National High Technology Research and Development Program of China (2006AA02Z309), and JSPS-NSFC collaboration project.

\section{References}

1. Eisenberg, D., Marcotte, E., Xenarios, I., Yeates, T.: Protein function in the postgenomic era. Nature 405, 823-826 (2000)

2. Itzhaki, Z., Akiva, E., Altuvia, Y., Margalit, H.: Evolutionary conservation of domain-domain interactions. Genome Biology 7(12), R125 (2006)

3. Zhao, X., Wang, Y., Chen, L., Aihara, K.: Protein domain annotation with integration of heterogeneous information sources. Proteins: Structure, Function, and Bioinformatics (in press, 2008)

4. Sprinzak, E., Margalit, H.: Correlated sequence-signatures as markers of proteinprotein interaction. J. Mol. Biol. 311(4), 681-692 (2001)

5. Deng, M., Mehta, S., Sun, F., Chen, T.: Inferring domain-domain interactions from protein-protein interactions. Genome Res. 12(10), 1540-1548 (2002)

6. Liu, Y., Liu, N., Zhao, H.: Inferring protein-protein interactions through highthroughput interaction data from diverse organisms. Bioinformatics 21(15), 3279 $3285(2005)$

7. Riley, R., Lee, C., Sabatti, C., Eisenberg, D.: Inferring protein domain interactions from databases of interacting proteins. Genome Biol. 6(10) (2005)

8. Xenarios, I., Rice, D., Salwinski, L., Baron, M., Marcotte, E.M., et al.: DIP: the Database of Interacting Proteins. Nucl. Acids Res. 28(1), 289-291 (2000)

9. Guimaraes, K., Jothi, R., Zotenko, E., Przytycka, T.: Predicting domain-domain interactions using a parsimony approach. Genome Biology 7(11), R104 (2006)

10. Lee, H., Deng, M., Sun, F., Chen, T.: An integrated approach to the prediction of domain-domain interactions. BMC Bioinformatics 7(1), 269 (2006)

11. Zhao, X., Li, X., Chen, L., Aihara, K.: Protein classification with imbalanced data. Proteins: Structure, Function, and Bioinformatics 70(4), 1125-1132 (2008)

12. Zhao, X., Chen, L., Aihara, K.: Gene function prediction using labeled and unlabeled data. BMC. Bioinformatics 9, 57 (2008)

13. Qi, Y., Bar-Joseph, Z., Klein-Seetharaman, J.: Evaluation of different biological data and computational classification methods for use in protein interaction prediction. Proteins: Structure, Function, and Bioinformatics 63(3), 490-500 (2006)

14. Tong, A., Lesage, G., Bader, G., Ding, H., Xu, H., et al.: Global Mapping of the Yeast Genetic Interaction Network. Science 303(5659), 808-813 (2004)

15. Weston, J., Perez-Cruz, F., Bousquet, O., Chapelle, O., Elisseeff, A., et al.: Feature selection and transduction for prediction of molecular bioactivity for drug design. Bioinformatics 19(6), 764-771 (2003)

16. Bateman, A., Coin, L., Durbin, R., Finn, R., Hollich, V., et al.: The Pfam protein families database. Nucl. Acids Res. 32(suppl.1), D138-141 (2004)

17. Raghavachari, B., Tasneem, A., Przytycka, T., Jothi, R.: DOMINE: a database of protein domain interactions. Nucl. Acids Res. 36(suppl.1), D656-661 (2008) 
18. Stein, A., Russell, R., Aloy, P.: 3did: interacting protein domains of known threedimensional structure. Nucl. Acids Res. 33(suppl.1), D413-417 (2005)

19. Finn, R., Marshall, M., Bateman, A.: iPfam: visualization of protein-protein interactions in PDB at domain and amino acid resolutions. Bioinformatics 21(3), 410-412 (2005)

20. Raja, J., Praveen, F., Asba, T., Teresa, M.: Co-evolutionary analysis of domains in interacting proteins reveals insights into domain-domain interactions mediating protein-protein interactions. Journal of Molecular Biology 362, 861-875 (2006) 